\title{
Open cluster kinematics with Gaia DR2 ${ }^{\star}$ (Corrigendum)
}

\author{
C. Soubiran ${ }^{1}$, T. Cantat-Gaudin ${ }^{2}$, M. Romero-Gómez ${ }^{2}$, L. Casamiquela ${ }^{1}$, C. Jordi ${ }^{2}$, A. Vallenari ${ }^{3}$, T. Antoja ${ }^{2}$,
} L. Balaguer-Núñez ${ }^{2}$, D. Bossini ${ }^{3}$, A. Bragaglia ${ }^{4}$, R. Carrera ${ }^{3}$, A. Castro-Ginard ${ }^{2}$, F. Figueras ${ }^{2}$, U. Heiter ${ }^{6}$, D. Katz ${ }^{7}$, A. Krone-Martins ${ }^{5}$, J.-F. Le Campion ${ }^{1}$, A. Moitinho ${ }^{5}$, and R. Sordo ${ }^{3}$

\footnotetext{
${ }^{1}$ Laboratoire d'Astrophysique de Bordeaux, Univ. Bordeaux, CNRS, B18N, allée Geoffroy Saint-Hilaire, 33615 Pessac, France e-mail: caroline.soubiran@u-bordeaux.fr

2 Institut de Ciències del Cosmos, Universitat de Barcelona (IEEC-UB), Martí i Franquès 1, 08028 Barcelona, Spain

3 INAF-Osservatorio Astronomico di Padova, vicolo Osservatorio 5, 35122 Padova, Italy

4 INAF-Osservatorio di Astrofisica e Scienza dello Spazio, via Gobetti 93/3, 40129 Bologna, Italy

5 CENTRA, Faculdade de Ciências, Universidade de Lisboa, Ed. C8, Campo Grande, 1749-016 Lisboa, Portugal

6 Department of Physics and Astronomy, Uppsala University, Box 516, 75120 Uppsala, Sweden

7 GEPI, Observatoire de Paris, Université PSL, CNRS, 5 Place Jules Janssen, 92190 Meudon, France
}

A\&A, 619, A155 (2018), https://doi .org/10.1051/0004-6361/201834020

Key words. stars: kinematics and dynamics - Galaxy: kinematics and dynamics - open clusters and associations: general errata, addenda

Due to an unfortunate error in the computation of distance between clusters, Tables 4 and 5, and Fig. 12 in Sect. 3.3 of the original paper contain incorrect values. Here we present the updated tables and figure.

Table 4 gives the list of cluster pairs that differ by less than $100 \mathrm{pc}$ in distance and $5 \mathrm{~km} \mathrm{~s}^{-1}$ in velocity in our high-quality sample. The closest pair includes ASCC 16 and ASCC 21, which are separated by $\sim 13 \mathrm{pc}$, with a velocity difference of $4.5 \mathrm{~km} \mathrm{~s}^{-1}$. RSG 7 and RSG 8, as well as ASCC 16 and ASCC 19, are also close pairs separated by $\sim 24 \mathrm{pc}$, with velocity differences of $2.3 \mathrm{~km} \mathrm{~s}^{-1}$ and $3.6 \mathrm{~km} \mathrm{~s}^{-1}$ respectively, thus good candidates to be physically related. Several possibly larger complexes may be found in that table, formed by clusters that appear more than one time. Table 5 gives a list of candidates binaries from the literature. The most famous binary cluster formed by $h$ and $\chi$ Persei (NGC 869 and NGC 884, Messow \& Schorr 1913) appears to have a separation of $19.5 \mathrm{pc}$. An excellent candidate binary is the pair Collinder 394 and NGC 6716, which lie at a distance of $\sim 11$ pc from each other. IC 2602 and Platais 8 are also close from each other $(\sim 35 \mathrm{pc})$ and they are possibly physically related, owing to their common age in DAML and similar velocity (also reported in Table 4). The other pairs in Table 5 have much larger separation and they are more likely to result from a chance alignment than that they are a physical binary system.
Table 4. Pairs of OCs differing by less than $100 \mathrm{pc}$ in their Galactic position and $5 \mathrm{~km} \mathrm{~s}^{-1}$ in velocity in the high-quality sample.

\begin{tabular}{llrr}
\hline \hline Cluster 1 & Cluster 2 & $\Delta$ pos $(\mathrm{pc})$ & $\Delta V\left(\mathrm{~km} \mathrm{~s}^{-1}\right)$ \\
\hline ASCC 105 & Roslund 5 & 85.1 & 3.7 \\
ASCC 127 & RSG 7 & 61.8 & 3.3 \\
ASCC 127 & RSG 8 & 81.9 & 2.8 \\
ASCC 16 & ASCC 19 & 24.6 & 3.6 \\
ASCC 16 & ASCC 21 & 13.2 & 4.5 \\
ASCC 19 & NGC 2232 & 96.0 & 3.9 \\
ASCC 58 & Alessi 5 & 99.4 & 3.9 \\
ASCC 58 & BH 99 & 54.8 & 3.3 \\
ASCC 97 & IC 4725 & 84.0 & 3.8 \\
Alessi 20 & Stock 12 & 59.3 & 2.2 \\
Alessi 5 & BH 99 & 50.5 & 3.6 \\
Collinder 135 & Collinder 140 & 84.6 & 3.6 \\
Collinder 135 & NGC 2451B & 69.2 & 2.4 \\
Collinder 140 & NGC 2451B & 50.8 & 1.8 \\
Collinder 69 & Gulliver 6 & 83.4 & 3.5 \\
Gulliver 20 & IC 4665 & 96.3 & 2.7 \\
IC 2391 & Platais 9 & 43.9 & 1.6 \\
IC 2602 & Platais 8 & 35.9 & 4.3 \\
NGC 2451B & NGC 2547 & 82.7 & 3.4 \\
RSG 7 & RSG 8 & 24.3 & 2.3 \\
Stock 23 & Trumpler 3 & 70.1 & 2.7 \\
\hline
\end{tabular}

\footnotetext{
* The table with clusters velocities is only available at the CDS via anonymous ftp to cdsarc. u-strasbg. fr (130.79.128.5) or via http://cdsarc.u-strasbg.fr/viz-bin/qcat?J/A+A/623/C2
} 

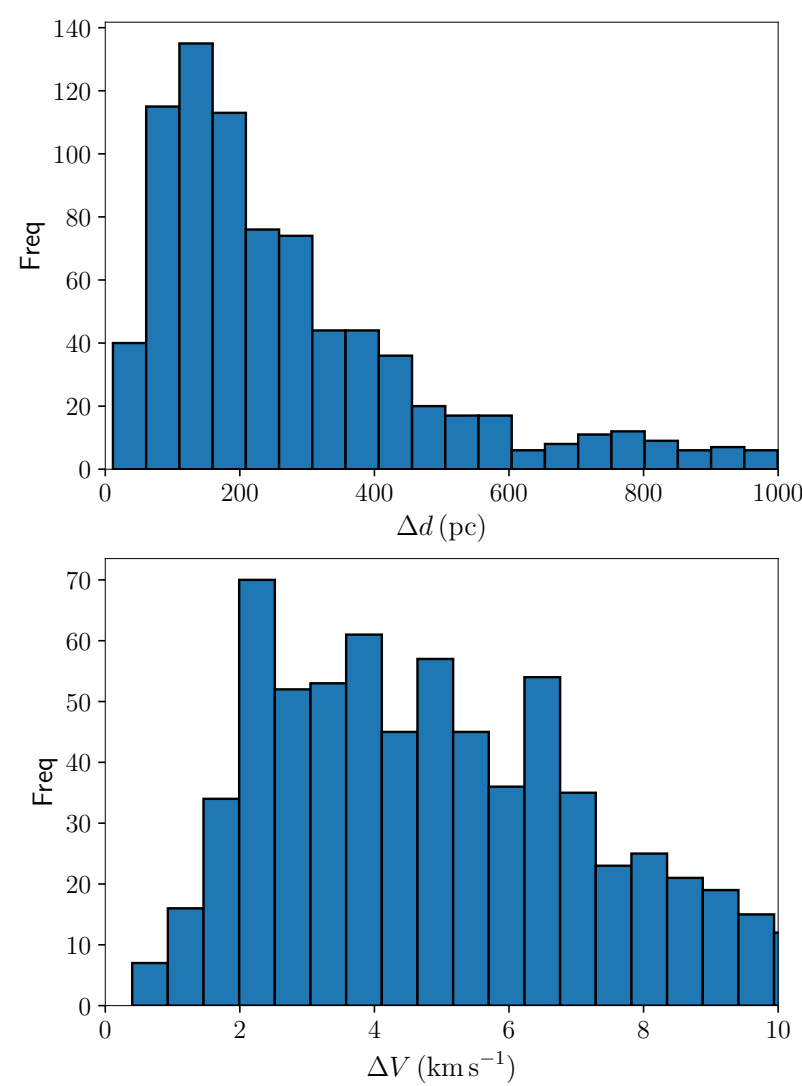

Fig. 12. Histogram of the distance between nearest neighbours in the high-quality sample (upper panel) and the same in velocity (lower panel).
Table 5. Separation in space and velocity of binary candidates from the literature, computed from our mean parameters for 861 OCs.

\begin{tabular}{lllrr}
\hline \hline Cluster 1 & Cluster 2 & ref & $\Delta$ pos $(\mathrm{pc})$ & $\Delta V\left(\mathrm{~km} \mathrm{~s}^{-1}\right)$ \\
\hline Alessi 13 & Mamajek 1 & 1 & 92.5 & 5.1 \\
Alessi 21 & NGC 2422 & 1 & 120.8 & 9.3 \\
Platais 8 & IC 2602 & 1 & 35.9 & 4.3 \\
Turner 9 & ASCC 110 & 1 & 275.6 & 9.0 \\
Collinder 394 & NGC 6716 & 1 & 11.4 & 13.8 \\
IC 1396 & NGC 7160 & 1 & 87.6 & 13.9 \\
NGC 869 & NGC 884 & 2 & 19.5 & 19.9 \\
NGC 5617 & Trumpler 22 & 3 & 79.8 & 10.4 \\
IC 4756 & NGC 6633 & 4 & 82.9 & 8.0 \\
\hline
\end{tabular}

References. (1) Conrad et al. (2017), (2) Messow \& Schorr (1913), (3) De Silva et al. (2015), (4) Casamiquela et al. (2016).

\section{References}

Casamiquela, L., Carrera, R., Jordi, C., et al. 2016, MNRAS, 458, 3150 Conrad, C., Scholz, R.-D., Kharchenko, N. V., et al. 2017, A\&A, 600, A106 De Silva, G. M., Carraro, G., D'Orazi, V., et al. 2015, MNRAS, 453, 106 Messow, B., \& Schorr, R. R. E. 1913, Astronomische Abhandlungen der Hamburger Sternwarte, 2, 1 